\title{
Trainees forum
}

\section{"Psychiatrist, please see"}

\author{
Peter Flanigan, Registrar, Tyrone and Fermanagh Hospital, Omagh, Co. Tyrone, \\ Northern Ireland
}

\section{The assessment of the (para) suicidal}

Many junior doctors, some with only undergraduate training, will this August feel at the pointed end of psychiatry when they are asked to see an 'overdose'. The referral letter is unlikely to be more specific concerning what service they are to render to the patient or the referring physician. In the psychiatric textbooks the tyro will find a wealth of actuarial data but little clinical advice. After four years as a junior psychiatrist I present a personal philosophy of what one is doing when one assesses the survivor of a (para) suicidal act.

\section{Rationale}

The rationale is screening: to try to discern amongst the many people who take overdoses, for a great many motives, certain sub-groups whom one will try to help: those suffering from mental illness; those seeking help (the 'cry for help' syndrome) and those with a significantly increased suicidal risk.

The rest, I suggest, are scarcely the province of psychiatry but may yet fall within the scope of community medicine, for the world is currently experiencing a pandemic of parasuicidal acts which makes a significant demand on health resources and is suspected to claim more than few unintended lives.

Those suffering from mental illness should be offered treatment, if necessary, under the provisions of the Mental Health Act. Those seeking help should be counselled personally or directed to an appropriate agency, e.g. social services, AA, Cruse, CaraFriend, Pregnancy Counselling, AIDS counselling etc.

Because all who take overdoses or make other para suicidal acts are at an increased risk of suicide, it is something of an arbitrary judgement to decide who are at significantly increased risk; nor is their management straightforward. The law and ethical guidelines are vague about what should be done for people who are not mentally ill but have a substantial risk of selfinflicted serious physical harm. It is an offence to aid suicide and we all have a duty to prevent suicide. The psychiatrist who is seeing suicidal patients should, as a professional attitude, wholly reject suicide.
I cannot be any better than vague myself but the reader will note that the Mental Health Act permits a "detention for assessment" and that the employing Health Authority only, and not the individual doctors and nurses, is legally liable for damages resulting from a successful suit for wrongful detention.

\begin{abstract}
Assessment
The patient is assessed by history, corroborative history and mental state assessment leading to a formulation and diagnosis. One is looking in particular for risk factors predisposing to successful suicide. These can be grouped under five headings: factors in the attempt; the demographic data; the illness; the mental state; and additional factors in certain populations, e.g. adolescents and in-patients.
\end{abstract}

\section{Factors in the attempt}

Whether sober; long contemplated; preparations made in advance; measures taken to avoid discovery/ interruption; made Will or disposed of possessions; left suicide note; lethal method - especially if violent or painful.

\section{Demographic factors}

Completed suicide is higher in males; the elderly; divorced; unemployed; those living alone; with physical illness; with psychiatric illness; alcoholics; drug addicts; and those in professions with knowledge or access, e.g. pharmacists, dentists, doctors, Armed Forces.

\section{Factors in the illness}

Manic-depressive psychosis, especially in early years of the illness; schizophrenia; endogenous depression; alcoholism; family history; chronic depression; previous suicide attempts.

\section{Factors in the mental state}

Lengthy suicidal preoccupation; suicidal intent at the time of the act; not glad to have survived; expresses suicidal intent after resuscitation; hopelessness 
regarding the future; deeply depressed mood judged by consistency, severity, biological symptoms, despair, delusions, hallucinations, loss of judgement.

\section{Extra factors in certain populations}

In-patients: early stages of recovery from depression; on leave from hospital; recently discharged; recent suicidal modelling (by another patient); alienation (poor relations with staff, disillusioned with treatment).

Adolescents: males; after disciplinary action; recent school refusal; within two weeks of birthday; discussed, threatened or attempted suicide in previous 24 hours; aggressive and anti-social; without close friend or confidante.
Ideally this assessment should be discussed with one's supervising consultant and advice, help or treatment offered as indicated.

Lastly a word of comfort. The assessment of suicidal risk is recognised to be notoriously difficult. Suicide is thankfully a rare event. In those who are at high risk one seeks to invalidate one's own prediction. In those whom you treat it is impossible to know when you have averted a completed suicide. Also be reassured that most 'overdoses' have very few risk factors and cause their assessor little anxiety. The experienced psychiatrists I have spoken to reckon they admit $10 \%$ or less of the 'overdoses' they see. 